\title{
LOCAL TEXTURE MEASUREMENTS BY EBSP. NEW COMPUTER PROCEDURES
}

\author{
D. Juul Jensen and N.H. Schmidt \\ Materials Department, Ris $\varnothing$ National Laboratory, DK-4000 Roskilde, Denmark
}

\begin{abstract}
Two new computer procedures for analysis of electron back scattering patterns (EBSP) are presented. One is a semiautomatic procedure for on-line analysis of EBSPs. The other is an image processing procedure for computer identification of bands in an EBSP. These two procedures may be combined for fully automatic indexing of EBSPs.
\end{abstract}

\section{INTRODUCTION}

To further the understanding of the formation of textures, for example during thermomechanical processing, it is necessary to be able to correlate "macro textures" and microstructures. Thus, measurements of crystallographic orientations in selected local areas of the microstructure are needed. A very powerful technique for collecting this type of data over a large sample area is the electron back scattering pattern (EBSP) technique ${ }^{1}$, and the technique has already been extensively used for a wide range of applications - e.g. to determine misorientations between subcells in deformation structures, orientation relations between nuclei and deformation matrix, size/orientation relationships, two (or three) point lattice orientation correlations, grain boundary geometry (mesotextures) and grain growth. (For reviews see e.g. Adams ${ }^{2}$ and Juul Jensen and Randle $^{3}$ ). The greatest drawback of the EBSP, and other local texture techniques, to these types of investigations is the practical difficulty of obtaining sufficient data to achieve a statistically significant result in a reasonably time. Fast, user friendly - and if possible fully automatic - procedures for data acquisition and analysis are therefore needed. This was the aim of the present work.

\section{EXPERIMENTAL}

The standard JEOL EBSP set-up with a CCD very-low-light TV camera mounted in the rear port is used in a 840 SEM. The samples are mounted in a specially designed pre-tilted specimen holder (inclined at $20^{\circ}$ to the incident beam). From the TV camera the video signal is converted to a frame averaged digital image through a frame grabber. For further details on the experimental set-up see ref. ${ }^{4}$. A typical example of an EBSP from commercially pure aluminium is shown in Fig. 1a. 


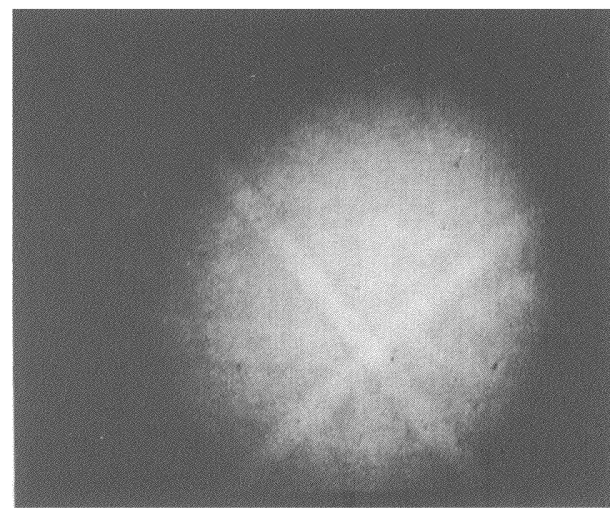

a)

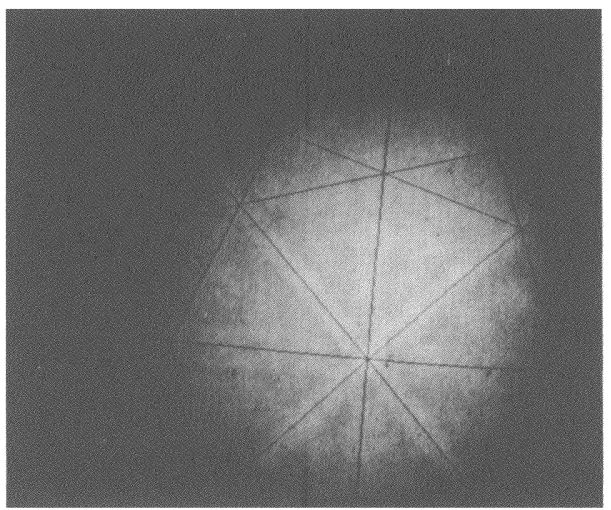

b)

Fig. 1. a) EBSP from aluminium. b) Simulated EBSP superimposed on the experimental pattern.

\section{EBSP-ANALYSIS PROCEDURE}

In the following section a new semi-automatic procedure for on- line determination of crystallographic orientations from EBSPs is described. As input the center line for at least 2 EBSP bands (lattice planes) have to be identified on the monitor using a computer generated rubber stick. No information about the crystallographic indices of the bands is needed.

A flow diagram showing the steps in the analysis procedure is given in Fig. 2. Before any EBSPs from a given sample material can be analysed a look-up table with interplanar angles characteristic of that sample material has to be created: Based on input information about the crystal symmetry and lattice spacing/angles for the given sample material the structure factors for the different reflecting lattice planes are calculated. The orientations of the strongest reflecting lattice planes are determined using standard matrix calculations. The interplanar angles between these are then calculated and stored in the look-up table. When the look-up table is stored, it is used for all further analysis of EBSPs of that material, i.e. look-up tables only have to be created once.

For each experimental EBSP, the position of two or more bands are identified by the user. These are transformed into a set of three dimensional planes and the corresponding interplanar angles are calculated. By comparing with the angles in the look-up table the values which give the best agreement with the experimental input are determined. The corresponding simulated EBSP is worked out and is superimposed on the experimental EBSP on the computer monitor. It is thereby easy to check if the simulated solution agrees with the experimental one. An example of a simulated EBSP superimposed on an experimental EBSP is shown in Fig. 1 b. 
START

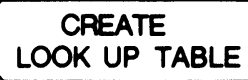

FOR EACH EBSP:

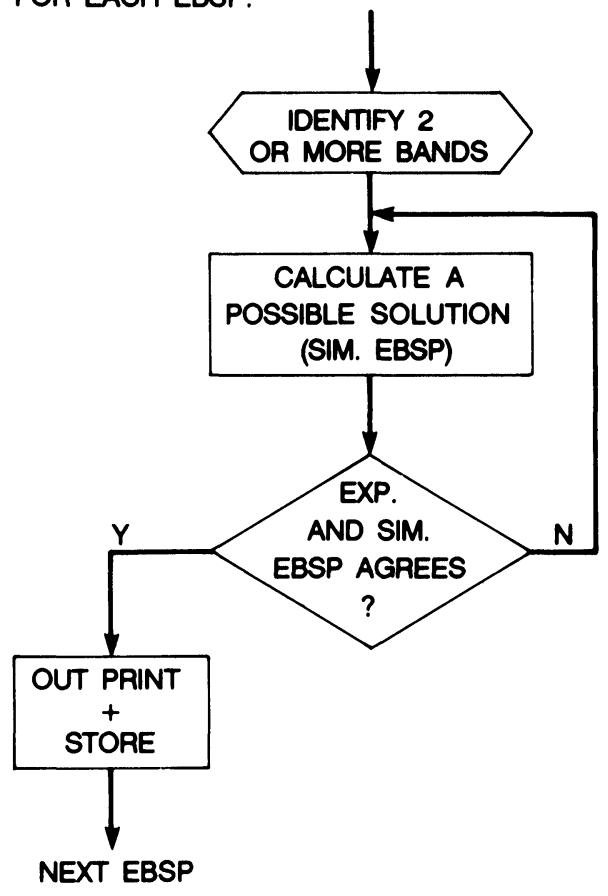

Fig. 2. Flow diagram for the analysis procedures.

If two bands are used as input and these two bands are not intersecting each other at a $90^{\circ}$ angle, typically 1 to 3 simulations (on average 1.4) are used to find the correct solution. When the angle is $90^{\circ}$, about 9 to 15 (on average 10.2) attempts are needed. If, instead, three input bands are used and these bands do not intersect in a common pole, the correct solution is found in the first attempt for $\sim 80 \%$ of the EBSPs. For the remaining 20\%, up to 5 simulations were suggested before the correct solution appeard. For further details see ref. ${ }^{4}$

\section{IMAGE PROCESSING PROCEDURE}

In the following section a procedure for automatic recognition of intense bands in EBSPs is described. With the present experimental set- up, the quality of the EBSPs are rather poor. Typically the maximum intensities within the bands are only 1-2\% larger than that in the surrounding background, there are no sharp edges between bands and the background intensity level varies significantly with position in the EBSP. Standard edge enhancement image processing procedures or line detection routines could not be used to identify the bands, therefore new EBSP dedicated routines were developed.

A flow diagram showing the steps in the image processing procedure is given in Fig. 3. First step is image enhancement: A global background correction is done by subtracting an EBSP without bands measured under identical experimental conditions from the actual EBSP. To further reduce the background noise the picture is smeared by a local average over $3 \times 3$ pixels. An example of an enhanced pattern is shown in Fig. 4a. The next step is simplification of the enhanced EBSP. This is done by scanning the pattern horizontally and finding the local maxima. If the maxima is accepted as a part 


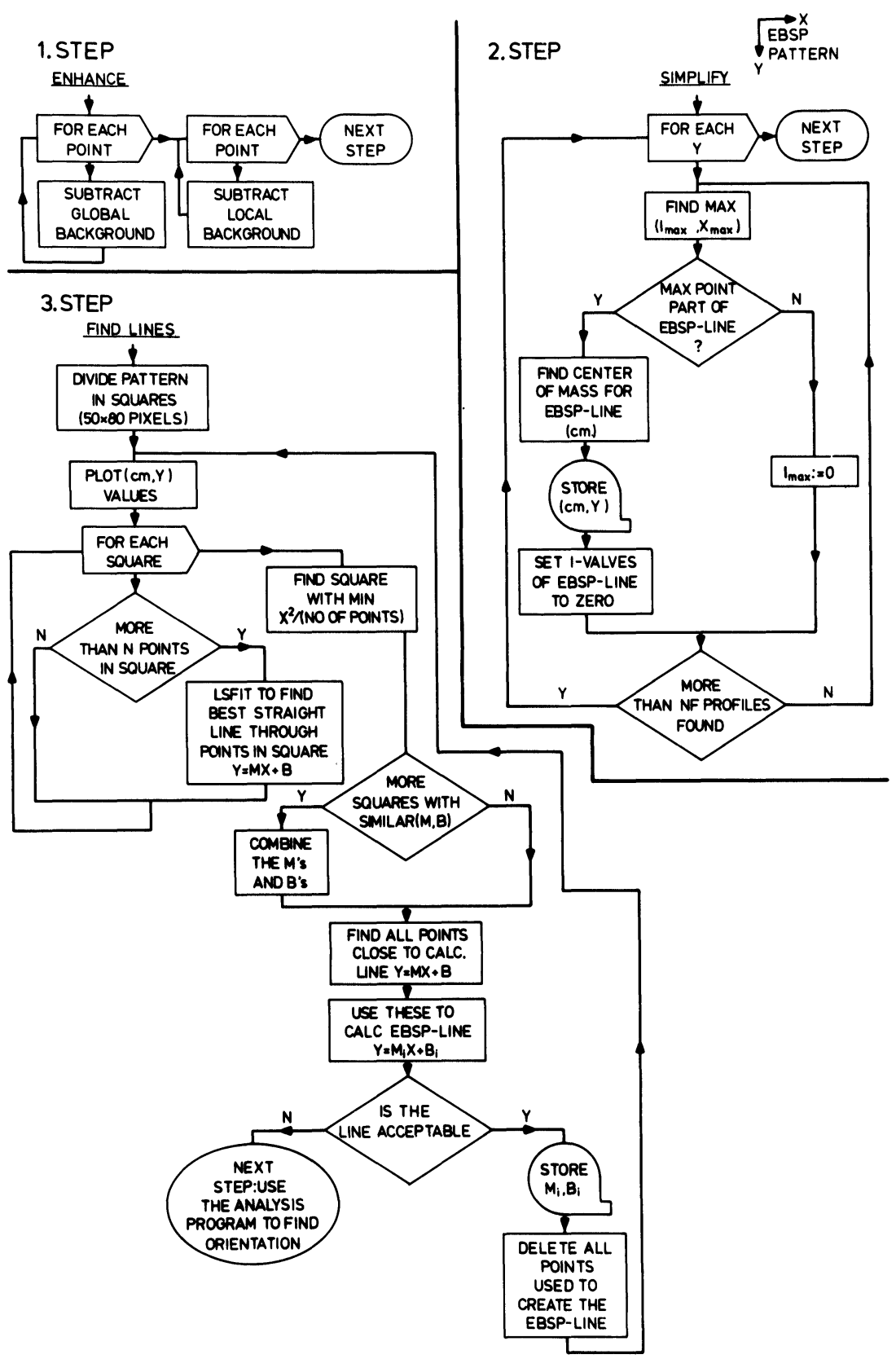

Fig. 3. Flow diagram for the image processing procedure. 
of an EBSP band, the position (x,y) is stored. Typically a few hundred points $(\mathrm{x}, \mathrm{y})$ are found in this way. An example of such a simplified pattern is shown in Fig. 4b, where bright spots show the selected points.

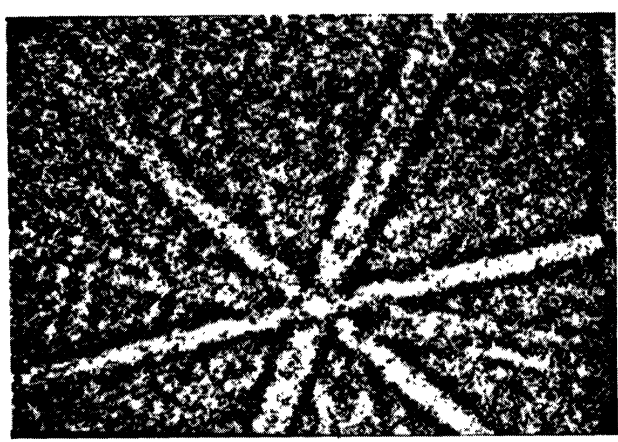

a)

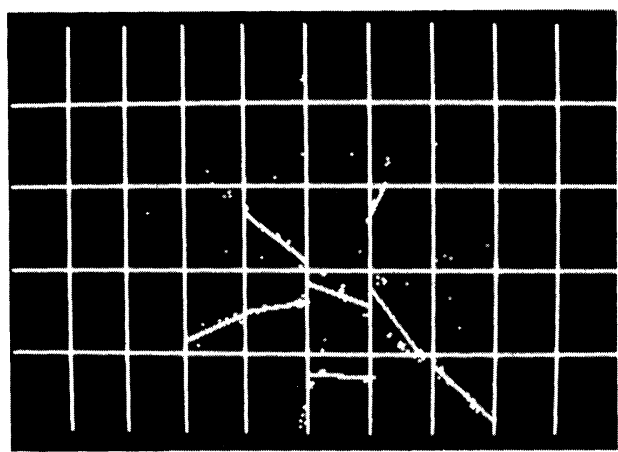

c)

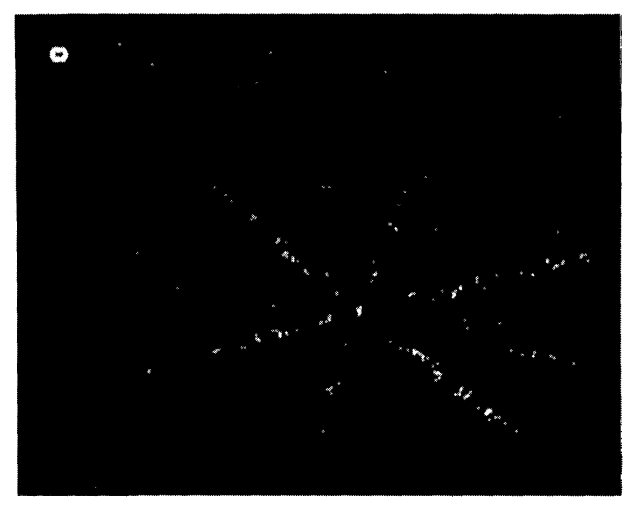

b)

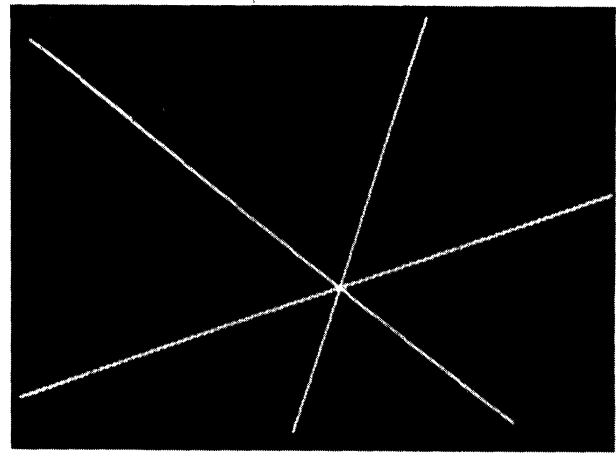

d)

Fig. 4. The various steps in the image processing procedure. a) the enhanced EBSP. b) the simplified EBSP. c) the simplified EBSP divided into squares. d) 3 center lines identified.

From this simplified pattern the EBSP center lines is to be determined. This means that the computer has to identify an unknown number of intersecting lines which is not a trivial matter considering the limited computer capacity of a PC. In the present procedure it is done by dividing the simplified pattern into a number of squares (typically 50) and then use a least squares fit (lsfit) routine to find the best straight lines through the points in each square. Clearly, a very bad fit between the lsfit line and the points is obtained for those squares containing intersecting EBSP lines (poles), i.e. the number of squares has to be selected carefully. An example of how a simplified pattern is divided into squares and the resulting lsfit lines is shown in Fig. 4c. The fitted slope and insection values ( $m$ and $b$ in the equation $y=m x+b$ ) for the squares which give a good fit between the lsfit line and points are then combined to identify a set of overall lines. These lines correspond to EBSP center lines. Typically 2-3 EBSP center lines are identified in this way. An example is shown in Fig. 4d. For further details see ref. ${ }^{6}$. 


\section{DISCUSSION}

The new analysis procedure described here can be compared to the more standard pole/zone axis analysis procedure ${ }^{1}$. The same principles are used for getting computer access to the EBSP, but the data acquisition is different. Using the new procedure the user only have to identify two EBSP-bands and no information about which crystal planes these bands represent is needed; i.e. the procedure is very easy to use. Further it is advantageous that it can be used for any crystal symmetry and that the visual inspection of the correspondence between a simulated and an experimental EBSP is very precise with the new routine.

The image processing procedure seems to be reliable. When a series of EBSPs for a given specimen has to be analysed,the first few patterns are used to set and check the calibration constants. Once these constants are set, they are used for the rest of the series. In general, at least two EBSP center lines are correctly identified by the computer, however in few cases (typically less than 5\%) only one line could be found. This relates to the relative poor quality of the EBSPs using the present experimental set-up, and at present we consider it more important to improve the quality of the pattern and thereby ease the computer line recognition than try to develop more sophisticated computer procedures.

The two computer procedures may be used together for fully automatic indexing of EBSPs. In this case, the image processing procedure is used to identify at least two EBSP bands, then the analysis procedure calculates possible solutions and at last a check routine finds the correct solution. In this check routine the correspondence between the simulated and experimental EBSPs is digitized. This is done by making a summation of the experimental intensities along the suggested EBSP bands (as determined by the analysis procedure); after a normalization, these values are compared. Typically the value, corresponding to the correct solution, is at least $10 \%$ higher than the others. If this is not the case, the experimental pattern has to be stored in the computer for later manual indexing. Also, if less than two lines are found by the image processing procedure, the EBSP has to be stored.

At present this combination of routines for fully automatic indexing of the EBSPs seems to work well but further tests are needed before it can be concluded if it is reliable enough to continue the development towards a fully automatic equipment for example with step motor/computer controlled sample movements.

\section{REFERENCES}

1. D.J. Dingley, Scan Electron Microscopy, 11, 569 (1984).

2. B. Adams, these proceedings.

3. D. Juul Jensen and V. Randle, In "Materials Architecture" (Bilde-Sørensen et al. eds., Risø 1989) p. 103.

4. N.H. Schmidt, J.B. Bilde-Sørensen and D. Juul Jensen, to be published.

5. "Spider, Subroutine Package For Image Data Enhancement and Recognition - Users Manual" (Agency Ind. Sci. Tech. eds, Japan 1983).

6. D. Juul Jensen and N.H. Schmdit, to be published in "Recrystallization '90". 